\title{
Evidência de validade de critério do Bender-Sistema de Pontuação Gradual
}

\author{
Adriana Cristina Boulhoça Suehiro \\ Acácia Aparecida Angeli dos Santos \\ Universidade São Francisco
}

\begin{abstract}
RESUMO
Este estudo teve por objetivo buscar evidências de validade para o Bender-Sistema de Pontuação Gradual (B-SPG). Participaram 237 crianças, ambos os sexos, idade média de 8,48 anos (DP=0,50), de segundas e terceiras séries de escolas públicas e particulares do estado de São Paulo. As nove figuras do Bender, desenhadas em transparência, foram aplicadas coletivamente em uma única sessão nas salas de aula. Os resultados permitiram a identificação de evidência de validade de critério por série, visto que houve nítida superioridade de desempenho para as crianças da terceira série em relação às da segunda série. Por ser um sistema novo de um teste psicológico bastante utilizado, sugere-se que outras pesquisas sejam feitas buscando identificar outras evidências de validade.
\end{abstract}

Palavras-chave: psicometria; habilidade viso-motora; avaliação psicológica; teste de Bender

\section{ABSTRACT \\ Evidence of criterion validity of the bender scoring system}

This study aimed to examine evidences for the validity of the Bender Gradual Scoring System Bender-Sistema de Pontuação Gradual (B-SPG). Participants were 237 children of both sexes, mean age 8,48 ( $\mathrm{SD}=0,50)$, from second- and third-grade in public and private schools. The nine Bender figures, projected by a transparency projector, were applied collectively in a single session. The criterion validity was demonstrated for each grade, third-grade students showed a performance clearly superior to second-grade students. The results suggest the need of further studies to investigate other evidences of validity.

Keywords: psychometrics; visual-motor; psychological assessment; Bender Test

O Teste de Bender foi desenvolvido por Lauretta Bender em 1938, baseado nas experiências de Max Wertheimer que visavam o estudo da gestalt visual. Utilizando-se basicamente dos mesmos materiais, a autora adaptou as figuras originais usadas pelo pesquisador e transformou-a em uma tarefa viso-motora que era proposta a seus pacientes. Assim, solicitava que as crianças atendidas por ela copiassem os modelos apresentados, ao invés de somente descrevê-los (Colorni, 1994; Machado, 1978; Santucci \& GalifretGranjon, 1968; Tosi, 1990).

De acordo com Sisto, Santos e Noronha (2004), o teste era inicialmente utilizado apenas com finalidade clínica e os protocolos eram avaliados de maneira qualitativa, não havendo um sistema de correção com atribuição de pontos, conforme a execução de cada um dos nove desenhos que o instrumento englobava. Posteriormente foram elaborados sistemas de correção que permitiam a quantificação da análise e rigor na consistência da avaliação.

Alguns sistemas de correção e pontuação das figuras do Bender foram rapidamente adotados por profissionais da área e também obtiveram maior impacto na geração de pesquisas. Entre os mais utilizados e pesquisados estão o de Santucci e Pêcheux, que propuseram um estudo genético da reprodução das figuras, o de Clawson, que identificou sinais e desvios na reprodução das figuras como indicadores de perturbação emocional. Finalmente destaca-se o de Koppitz, destinado à avaliação da maturidade neurológica visomotora (Machado, 1978; Sisto, Bueno \& Rueda, 2003; Sisto, Santos \& Noronha, 2004).

Sabe-se que a habilidade percepto-motora tem sido destacada como importante para a aprendizagem. Assim, os aspectos avaliados pelo Bender têm sido entendidos como requisitos para o desenvolvimento de 
habilidades acadêmicas e o bom desempenho escolar (Bandeira \& Hutz, 1994; Sisto, Santos \& Noronha, 2004, entre outros).

$\mathrm{O}$ teste de Bender tem sido especialmente utilizado na avaliação sensório-motora e detectação de problemas de aprendizagem. Ao lado disso, tem sido empregado também para o diagnóstico de perturbações emocionais, para a avaliação de sujeitos delinqüentes, como teste de inteligência e como medida de personalidade em adolescentes, bem como para a predição do desempenho escolar e determinação da necessidade de psicoterapia (Arrillaga, Eschebarria \& Goya, 1981; Bandeira \& Hutz, 1994; McCarron \& Horn, 1979; Pinelli Jr. \& Pasquali, 1991/1992; Sisto \& cols., 2003; Vance, Fuller \& Lester, 1986).

Embora existam vários sistemas de avaliação para a verificação das dificuldades específicas em crianças, Machado (1978) afirma que um dos mais utilizados em nosso meio é o Developmental Bender Test Scoring System, criado por Koppitz em 1963, e destinado à avaliação da maturidade neurológica da criança para o diagnóstico de lesão cerebral. O sistema de Koppitz teve sua origem em uma investigação com 1104 crianças, ambos os sexos, entre cinco e dez anos e onze meses, dentre as quais se encontravam também crianças excepcionais, com problemas emocionais, lesão cerebral, dificuldades de aprendizagem e deficiência mental.

Baseada no caráter evolutivo associado à aprendizagem das funções gestálticas viso-motoras, Koppitz $(1963,1975)$ construiu uma escala de maturação para as figuras de Bender. Nos estudos iniciais havia muitas categorias de erro, sendo que posteriormente foram mantidas somente aquelas que se mostraram estatisticamente significativas. Logo, o método de correção proposto pela autora ficou composto por 30 itens divididos entre as categorias de distorção de forma, integração, rotação e perseveração.

Faz-se necessário, entretanto, destacar que somente as irregularidades grosseiras das respostas dadas pelas crianças em cada uma dessas categorias são pontuadas, ou seja, consideradas presentes, não sendo considerados os desvios insignificantes. Nesse sentido, pontuações altas indicam um desempenho pobre, enquanto pontuações baixas são qualificadas como um bom desempenho. Quanto às qualidades psicométricas, os estudos referentes à precisão entre os avaliadores da escala maturacional geraram coeficientes de correlação entre 0,88 a 0,96 . Após quatro meses, os sujeitos foram novamente avaliados, obtendo-se índi- ces de correlações significativos entre 0,55 e 0,66 . De forma geral, a escala mostrou-se sensível para captar as diferenças no desenvolvimento percepto-motor de idade em idade, dos 6 aos 10 anos (Koppitz, 1963).

Muitas são as críticas dirigidas ao Teste Gestáltico Viso-Motor de Bender, tanto internacional quanto nacionalmente, especialmente no que concerne ao sistema de Koppitz. Os estudos recuperados, tanto no âmbito internacional quanto nacional, apontam discrepâncias entre os achados das pesquisas realizadas em contextos diversificados. Tais problemas têm levado vários autores estrangeiros (Chan, 2001; Neale \& McKay, 1985; Silvestre, Salaverry \& Gonzáles, 1995) e brasileiros (Bartholomeu, Rueda \& Sisto, 2005; Britto \& Santos, 1996; Pinelli Jr. \& Pasquali, 1991/1992; Sisto, Noronha \& Santos, 2004; Sisto, Santos \& cols., 2004; Suehiro \& Santos, 2005) a questionarem-no como sistema de avaliação, apontando para a necessidade de uma ampla revisão.

Estudos estrangeiros como o de Chan (2001), por exemplo, enfatizam a necessidade de padrões apropriados às crianças avaliadas, ao apontarem para dificuldades de comparação dos resultados do Bender com crianças chinesas de 4,5 a 8,5 anos, quando pontuadas segundo a classificação norte-americana. Problemas referentes à consideração da pontuação total do teste e à identificação de dificuldades em relação ao caráter discriminante de idades na maturação viso-motora foram verificados também nos estudos de Silvestre e cols. (1995) com crianças peruanas. Ao compararem 74 escolares nascidos em Lima $(150 \mathrm{~m})$ com 93 nascidos na cidade de Cerro de Pasco (4340 m) detectaram diferenças significativas na maturidade perceptomotora de crianças peruanas de 7 e 8 anos residentes em locais de diferentes altitudes, sendo que a diferença entre a idade cronológica e a idade visomotora foi mais evidente nas meninas do que nos meninos.

Entre os trabalhos nacionais encontram-se vários que estudaram os parâmetros psicométricos do sistema Koppitz. Algumas das principais pesquisas publicadas na literatura científica no Brasil aparecem descritas a seguir.

Os parâmetros psicométricos do Teste de Bender, segundo o sistema de avaliação desenvolvido por Koppitz, foram analisados por Pinelli Jr. e Pasquali (1991/1992), com base no método de análise fatorial. Os autores constataram que a estrutura obtida vai de encontro à do sistema proposto pela autora, uma vez que a estrutura multifatorial revelada contradiz a estrutura unifatorial pressuposta por Koppitz (1963). Além disso, afirmam que alguns itens do teste pouco 
contribuem na qualificação de um escore global por não serem discriminativos e sugerem a necessidade de uma nova organização dos itens, referindo-se especialmente à necessidade de aumento do número de itens em alguns fatores. Aspectos conflitantes, como o encontrado nesse estudo, mostram que o sistema de correção de Koppitz necessita ser reavaliado para que possa ser utilizado em diferentes culturas.

Bandeira e Hutz (1994), por exemplo, investigaram o grau de predição do rendimento escolar na primeira série por meio da aplicação dos testes Desenho da Figura Humana (DFH), Bender e Raven. Os resultados apontaram para correlações significativas entre os três testes e o rendimento, embora apenas o Bender e os itens evolutivos do DFH tenham contribuído para explicar a variância do rendimento escolar.

Os resultados da pesquisa realizada por Britto e Santos (1996) com 398 crianças do estado no Rio de Janeiro apontaram problemas nas pontuações alcançadas com a amostra estudada, quando se focalizavam variáveis relacionadas à idade e ao gênero. Os autores concluíram que era necessária cautela na utilização das normas de Koppitz aqui no Brasil.

Mais recentemente, Sisto, Noronha e cols. (2004) analisaram os critérios de distorção de forma relativos ao caráter evolutivo, proposto pelo Developmental Bender Test Scoring System, em uma amostra de 314 crianças brasileiras de ambos os sexos, com idades variando entre 7 e 10 anos, das séries iniciais do ensino fundamental. Com base nos achados dessa pesquisa, demonstraram a necessidade de uma revisão dos critérios da autora, uma vez que somente em três das seis medidas, as crianças mais velhas diminuíram as distorções nas figuras. Dessa forma, os autores consideraram que os indicadores referentes ao critério de deformação das figuras não discriminam as idades estudadas nem em cada figura per si, nem na soma de todas elas.

De forma semelhante, ao focalizarem o critério de integração, Sisto, Santos e cols. (2004) observaram que os resultados foram significativos para discriminar algumas figuras em relação às idades. Todavia, recorrendo ao teste de Tukey, verificaram que as idades não foram adequadamente diferenciadas em nenhuma das medidas, sendo que em cada uma delas e na pontuação total, apenas dois grupos etários foram formados. Além de não se ajustarem ao critério desenvolvimental previsto, os autores identificaram a existência de coeficientes de consistência interna baixos em cada uma das figuras, o que corrobora os achados do estudo de Pinelli Jr. e Pasquali (1991/ 1992), também realizado com crianças brasileiras.
Tais resultados reforçam, mais uma vez, a necessidade de uma revisão do critério proposto, uma vez que aparentemente ele está medindo coisas distintas.

As evidências de validade do Teste Gestáltico Viso-Motor de Bender foram investigadas com crianças do ensino fundamental, segundo o critério de distorção da forma e integração de Koppitz por Bartholomeu e cols. (2005). Os autores aplicaram o teste juntamente com a Escala de Avaliação de Dificuldades na Aprendizagem da Escrita (ADAPE) em 343 alunos, de ambos os sexos, de primeira a quarta séries do ensino fundamental de escolas públicas do interior do estado de São Paulo. Os principais resultados do estudo indicam que as pontuações do ADAPE discriminaram as séries, assim como algumas medidas de distorção e integração. No entanto, os autores verificaram que somente a medida de integração geral correlacionou-se com os problemas de escrita na terceira série. Os achados desse estudo se somam aos muitos outros, já mencionados, que no Brasil vêem apontando para a necessidade de uma reavaliação do sistema de correção de Koppitz.

Embora Kroeff (1992) tenha questionado a unidimensionalidade dos itens admitida por Koppitz como uma evidência de validade, identificou uma correlação positiva entre o desempenho no Bender e o nível sócio-econômico-cultural em mais de mil crianças. $\mathrm{O}$ autor concluiu que o sistema possibilita verificar que a escolaridade confere aos alunos uma maior competência viso-motora. Além disso, algumas pesquisas recuperadas também pontuam que as crianças mais velhas são muito mais propensas a terem melhor desempenho do que as mais novas, em função de uma tendência de melhor domínio da motricidade gráfica (Machado, 1978; Mazzeschi \& Lis, 1999; entre outros).

Em decorrência das controvérsias sobre o sistema de avaliação de habilidades viso-motoras mais utilizado com crianças (Noronha, 2002; Noronha \& cols., 2002) tem ocorrido o desenvolvimento de novas propostas alternativas, visando à superação das dificuldades detectadas. Nesse sentido, outros sistemas têm procurado superar as limitações identificadas com relação à interpretação das pontuações obtidas (La Puente \& Maciel Jr., 1984; Shapiro \& Simpson, 1995; Sisto, Santos \& cols., 2004).

Uma nova proposta de correção e interpretação dos escores obtidos pela população infantil é o BenderSistema de Pontuação Gradual (B-SPG) desenvolvido por Sisto, Noronha e Santos (2005). Trata-se de um sistema que diferencia as crianças quanto à gravidade 
dos erros cometidos, atribuindo uma pontuação gradual a cada item, conforme a presença de desvios em cada uma das figuras do Teste Gestáltico Viso-Motor de Bender. Essa nova proposta difere, por exemplo, da de Koppitz (1989), que atribui, independentemente da gravidade do erro, um ponto para as crianças que os cometem em relação à rotação, perseveração, distorção de forma e integração.

Utilizando o B-SPG, Suehiro e Santos (2005) desenvolveram um estudo com 287 crianças entre 7 a 10 anos, utilizando a Escala de Avaliação de Dificuldades na Aprendizagem da Escrita (ADAPE) para identificar eventuais diferenças entre alunos de $2^{\mathrm{a}}$ e $3^{\mathrm{a}}$ série de escolas públicas e particulares. Os resultados indicaram diferenças significativas entre as crianças que não apresentavam indícios de dificuldades de aprendizagem na escrita $(n=146)$ e as que apresentavam dificuldades média ou acentuada, conforme classificação do ADAPE ( $\mathrm{n}=40)$.

Alguns estudos já realizados têm indicado que esse novo sistema se apresenta como uma alternativa promissora para a avaliação da maturidade perceptomotora. Partindo desse pressuposto, o presente estudo teve por objetivo buscar evidência de validade de critério para o Bender-Sistema de Pontuação Gradual $(B-S P G)$ pela comparação de crianças em diferentes níveis de escolaridade e também por idade.

\section{MÉTODO}

\section{Participantes}

Participaram deste estudo 237 crianças, ambos os sexos, entre 8 e 9 anos $(M=8,48 ; D P=0,501)$, das segundas $(57 \% ; N=135)$ e terceiras séries $(43 \%$; $N=102)$, duas salas de cada série. Considerando o total da amostra, $123(51,9 \%)$ crianças eram do gênero masculino e $114(48,1 \%)$ do feminino, sendo que 137 $(57,8 \%)$ estudantes pertenciam à escola particular e $100(42,2 \%)$ à pública. Foi perguntado à professora das turmas se havia crianças que apresentavam alguma alteração perceptual importante que seria impeditiva para a realização das atividades propostas. Nesses casos o protocolo foi assinalado e posteriormente excluído.

\section{Material}

Foram utilizados neste estudo: (a) um questionário de identificação, no qual as crianças informaram os seguintes aspectos: nome, idade, sexo, série e instituição de ensino à qual pertenciam e (b) o Teste Gestálti- co Viso-Motor de Bender, que consiste de nove figuras (A, 1, 2, 3, 4, 5, 6, 7 e 8), desenhadas em transparência, para serem copiadas em uma folha em branco, sem qualquer tipo de auxílio mecânico. Conforme o Bender-Sistema de Pontuação Gradual (B-SPG) desenvolvido por Sisto e cols. (2005) foram pontuados os desvios em cada uma das figuras do teste. Aos protocolos, cujo avaliador não percebeu qualquer desvio relacionado à distorção da forma, não foi atribuído ponto algum e à medida que os desvios apareceram foram atribuídos de um a três pontos para a Figura $6 \mathrm{e}$ de um a dois pontos para as demais figuras, dependendo da severidade do erro, totalizando 21 pontos possíveis.

\section{Procedimento}

Após a aprovação do projeto pelo Comitê de Ética e a entrega do Termo de Consentimento Livre e Esclarecido às coordenadoras das escolas para que os pais das crianças consentissem em sua participação na pesquisa, os instrumentos foram aplicados coletivamente, em uma única sessão para cada sala, em horário de aula previamente cedido pelo professor. Inicialmente, as crianças preencheram as questões de identificação e, em seguida, foram solicitadas a copiarem, da melhor maneira possível, as figuras desenhadas em transparência que lhes foram apresentadas por meio de um retroprojetor. Foram necessários 30 minutos para que todo o procedimento planejado fosse executado.

\section{RESULTADOS}

Os resultados foram analisados em função dos objetivos pretendidos e foram utilizadas estatísticas descritivas e inferenciais para o tratamento dos dados obtidos. A Figura 1 apresenta a distribuição das pontuações de todos os participantes no $B-S P G$.

Como pôde ser observado, as crianças obtiveram uma média de 8,11 erros ( $D P=3,24)$, com uma pontuação mínima de 0 e máxima de 17 pontos no Bender. A pontuação mais freqüente foi 7 , sendo que $50 \%$ dos participantes obtiveram pontuação abaixo de 8 pontos. Os resultados obtidos e a distribuição das frequiências dispostas na Figura 1 evidenciam uma tendência de pontuações entre 4 e 12 pontos.

Buscou-se, ainda, verificar se o Bender-Sistema de Pontuação Gradual (B-SPG) seria sensível à captação de diferenças em razão da escolaridade das crianças avaliadas. As médias, desvios-padrão, pontuações mínimas e máximas por série podem ser observadas na Tabela 1. 


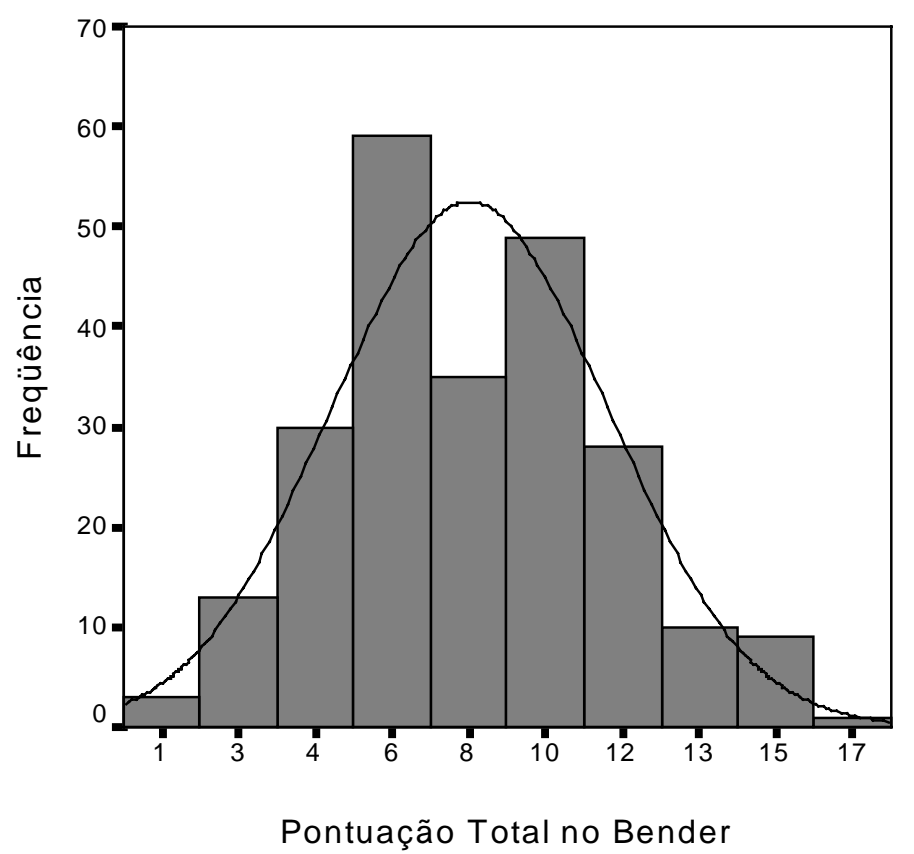

Figura 1. Freqüências das pontuações obtidas no Bender-Sistema de Pontuação Gradual.

Tabela 1. Pontuações mínimas, máximas, médias e desvios-padrão quanto à pontuação total obtida no BenderSistema de Pontuação Gradual por série.

\begin{tabular}{cccccc}
\hline Série & N & Mínimo & Máximo & Média & Desvio-Padrão \\
\hline Segunda Série & 135 & 2 & 17 & 8,90 & 3,33 \\
Terceira Série & 102 & 0 & 15 & 7,07 & 2,80 \\
\hline
\end{tabular}

Por estes resultados podem-se observar os escores obtidos pelas crianças de ambas as séries. A média e desvio-padrão das da terceira série são inferiores às da segunda, o que no caso do B-SPG aponta para um desenho mais parecido com a cópia fornecida. Com base no teste $t$ de Student contatou-se diferença estatisticamente significativa entre as séries analisadas, com superioridade de desempenho para os alunos da terceira série $(p<0,001)$.

Considerando os resultados obtidos, julgou-se relevante aprofundar as análises realizadas para verificar diferenças no desempenho das crianças para cada uma das nove figuras do Teste Gestáltico Viso-Motor de Bender. Tais resultados podem ser visualizados na Tabela 2.

Tabela 2. Comparação entre 0 desempenho das crianças de segunda e terceira série quanto à pontuação obtida e os valores de $t e p$ para cada uma das nove figuras do instrumento utilizado

\begin{tabular}{|c|c|c|c|c|c|c|}
\hline \multirow{2}{*}{ Figuras } & \multicolumn{2}{|c|}{ Segunda Série } & \multicolumn{2}{|c|}{ Terceira Série } & \multirow{2}{*}{$t$} & \multirow{2}{*}{$p$} \\
\hline & Média & $D P$ & Média & $D P$ & & \\
\hline$A$ & 0,48 & 0,530 & 0,22 & 0,413 & 4,189 & 0,000 \\
\hline 1 & 0,43 & 0,758 & 0,52 & 0,920 & $-0,825$ & 0,410 \\
\hline 2 & 1,29 & 0,558 & 1,09 & 0,631 & 2,590 & 0,010 \\
\hline 3 & 1,79 & 0,648 & 1,50 & 0,793 & 3,125 & 0,002 \\
\hline 4 & 0,94 & 0,570 & 0,89 & 0,673 & 0,601 & 0,549 \\
\hline 5 & 0,55 & 0,569 & 0,59 & 0,569 & $-0,537$ & 0,592 \\
\hline 6 & 1,60 & 0,784 & 1,25 & 0,909 & 3,132 & 0,002 \\
\hline $7 \mathrm{~A}$ & 0,72 & 0,729 & 0,52 & 0,641 & 2,189 & 0,030 \\
\hline $7 \mathrm{~B}$ & 0,64 & 0,728 & 0,44 & 0,606 & 2,285 & 0,023 \\
\hline 8 & 0,56 & 0,788 & 0,21 & 0,474 & 4,055 & 0,000 \\
\hline
\end{tabular}


Conforme pode ser observado, as crianças da terceira série apresentaram um melhor desempenho em todas as figuras, exceto nas figuras 1 e 5 , nas quais obtiveram uma média de erros mais elevada em comparação às crianças da segunda série $(M=0,52$ e $M=0,59$, respectivamente). Apenas nas figuras 4 e 5 não foram constatadas diferenças significativas entre as séries e, independentemente dessa variável, houve uma média de erros maior e, portanto, mais dificuldade na reprodução das figuras 2, 3, 4 e 6 .

Com relação à faixa etária dos sujeitos aqui estudados observou-se que estão dentro dos padrões etári- os esperados para cada uma das etapas de escolaridade estudadas. Assim a média de idade dos alunos da segunda série é de 8,16 anos $(D P=0,60)$ e da terceira série é de 9,17 anos $(D P=0,54)$. Considerando-se que o manual do Bender-Sistema de Pontuação Gradual $(B-S P G)$ traz nas suas normas, uma padronização da média de erros por faixa etária, julgou-se pertinente verificar se as crianças, aqui estudadas, apresentavam diferenças significativas em relação ao escore esperado para suas idades. Assim, utilizou-se o teste $t$ onesample para tal comparação. Os resultados obtidos estão apresentados na Tabela 3.

Tabela 3. Comparação entre o desempenho das crianças de oito e nove anos quanto à pontuação obtida e os valores de $t$ e $p$ para cada uma das nove figuras do BenderSistema de Pontuação Gradual

\begin{tabular}{ccccccc}
\hline \multirow{2}{*}{ Figuras } & \multicolumn{2}{c}{ Oito Anos } & \multicolumn{2}{c}{ Nove Anos } & \multirow{2}{*}{$\boldsymbol{t}$} & $\boldsymbol{p}$ \\
\cline { 2 - 5 } & Média & $D P$ & Média & $D P$ & & \\
\hline A & 0,44 & 0,530 & 0,28 & 0,453 & 2,492 & 0,013 \\
1 & 0,32 & 0,739 & 0,63 & 0,898 & $-2,872$ & 0,004 \\
2 & 1,27 & 0,586 & 1,13 & 0,605 & 1,723 & 0,086 \\
3 & 1,78 & 0,645 & 1,54 & 0,791 & 2,596 & 0,010 \\
4 & 0,93 & 0,558 & 0,91 & 0,676 & 0,198 & 0,843 \\
5 & 0,51 & 0,563 & 0,63 & 0,570 & $-1,633$ & 0,104 \\
6 & 1,55 & 0,800 & 1,35 & 0,904 & 1,836 & 0,068 \\
7A & 0,70 & 0,698 & 0,56 & 0,694 & 1,592 & 0,113 \\
7B & 0,56 & 0,690 & 0,55 & 0,681 & 0,178 & 0,859 \\
8 & 0,49 & 0,727 & 0,32 & 0,645 & 1,935 & 0,054 \\
\hline
\end{tabular}

Os dados obtidos demonstraram que as crianças do presente estudo apresentaram desempenho bastante similar ao da amostra. Assim, não foram detectadas diferenças significativas entre os escores das crianças da série respectiva e a idade da amostra de padronização.

\section{DISCUSSÃO}

O Teste Gestáltico Viso-Motor de Bender, mundialmente conhecido, têm sido largamente empregado na avaliação infantil e considerado como um instrumento útil para o diagnóstico diferencial de crianças com problemas de aprendizagem, uma vez que os aspectos avaliados pelo teste são concebidos como um requisito para o desenvolvimento de habilidades acadêmicas e um bom desempenho escolar (Arrillaga\& cols., 1981; Bandeira \& Hutz, 1994; McCarron \& Horn, 1979; Suehiro \& Santos, 2005; Vance \& cols., 1986).

Há anos que muitos estudiosos têm se referido a problemas de discriminação da habilidade visomotora do sistema Koppitz que têm sido detectados em estudos estrangeiros (Chan, 2001; Neale \& McKay, 1985; Silvestre \& cols., 1995), referindo-se ao seu poder restrito de aplicação fora do local onde foi desenvolvido. Também estudos nacionais apontam críticas contundentes ao referido sistema (Britto \& Santos, 1996; Kroeff, 1992; Sisto, Noronha \& cols., 2004; Sisto, Santos \& cols., 2004), sugerindo a necessidade de sua revisão.

Vale ressaltar a importante questão psicométrica apontada por Pinelli Jr. e Pasquali (1991/1992) em relação à principal evidência de validade do sistema de avaliação desenvolvido por Koppitz para o Teste de Bender. Os autores demonstraram a inexistência da estrutura unifatorial pressuposta pela autora quando da proposição de seus critérios em 1963. Importante lembrar, ainda, outro aspecto de invalidade identificado pelos autores, relacionados a itens que não discriminavam suficientemente a habilidade viso-motora que pretendiam medir. No estudo, aqui proposto, poucas figuras (1, 4 e 5) não demonstraram diferenças significativas favoráveis a um melhor desempenho de crianças da terceira série, conforme esperado. Tal 
aspecto merece ser mais explorado em pesquisas futuras com o sistema.

Tendo em vista essas questões, tomou-se como referência uma nova proposta de aplicação, pontuação e interpretação dos escores obtidos pela população infantil, o $B-S P G$, desenvolvido por Sisto e cols. (2005). Constatou-se que as crianças da terceira série apresentaram melhor desempenho no Bender quando comparadas às da segunda série, bem como diferenças estatisticamente significativas entre as séries analisadas, conforme o esperado.

Estes resultados permitiram a identificação de uma evidência de validade de critério para o BenderSistema de Pontuação Gradual (B-SPG) pela diferença de pontuação em razão da série frequientada pelas crianças da amostra focalizada, o que possibilita considerá-lo como um instrumento válido para a avaliação do desenvolvimento percepto-motor e sua possível associação com o progresso nas etapas sucessivas de escolarização. Tais achados estão em consonância com o esperado de um teste que seja válido para avaliar a habilidade percepto-motora de crianças, que deveria aumentar à medida que avancem nas etapas de escolaridade e também como preditivo de desempenho escolar (Bandeira \& Hutz, 1994; Kroeff, 1992; Suehiro \& Santos, 2005).

Além disso, algumas pesquisas recuperadas na literatura também pontuavam que as crianças mais velhas são muito mais propensas a terem melhor desempenho do que as mais novas, em função de uma tendência de melhor domínio da motricidade gráfica (Machado, 1978; Mazzeschi \& Lis, 1999; entre outros), o que corrobora os achados do presente estudo em relação à diminuição das distorções realizadas pelas crianças de nove anos avaliadas.

\section{CONCLUSÕES}

Diante dos resultados obtidos na presente pesquisa é possível afirmar-se que o $B-S P G$ pode ser utilizado como um instrumento de avaliação sensível para discriminar o incremento no desenvolvimento atribuído à escolaridade. Os resultados deste estudo confirmam a existência de diferenças significativas entre as crianças das duas séries escolares analisadas.

Observou-se ainda que a utilização de um teste, da forma como proposto no sistema $B-S P G$, torna-o diferente do restante das atividades comumente associadas ao contexto escolar e, mais especificamente, às atividades propostas pelos professores em sala de aula. Assim, seu caráter lúdico (cópia de figuras), a rapidez com que é aplicado, e a possibilidade de sua aplicação coletiva tornam o $B-S P G$ uma alternativa interessante para a identificação de possíveis problemas no desenvolvimento percepto-motor que possam estar associados ao desempenho escolar.

A aplicação coletiva por meio de transparências, autorizada pelo $B-S P G$, facilita a utilização do Bender como instrumento de avaliação percepto-motora no contexto escolar. Ao lado disso, a atribuição de uma pontuação gradual torna o sistema mais sensível para a captação de diferenças de desempenho mais sutis e oportuniza uma avaliação mais apropriada desse aspecto do desenvolvimento infantil. Todavia, há que se considerar que outras evidências de validade devem ser pesquisadas a fim de ampliar o conhecimento sobre as características psicométricas do sistema e contribuir para o aprimoramento dos instrumentos e procedimentos técnicos empregados na avaliação psicológica.

\section{REFERÊNCIAS}

Arrillaga, S. G. P., Eschebarria, C. C. \& Goya, I. O. (1981). Una investigación sobre aspectos intelectuales en una población de deficientes de un grupo marginal. Psiquis: Revista de Psiquiatria, Psicología y Psicosomática, 2 (1), 12-22.

Bandeira, R. D. \& Hutz, C. S. (1994). A contribuição dos testes DFH, Bender e Raven na predição do rendimento escolar na primeira série. Psicologia: Teoria e Pesquisa, 10 (1), 59-72.

Bartholomeu, D., Rueda, F. J. M. \& Sisto, F. F. (2005). Teste de Bender e dificuldades de aprendizagem: quão válido é o sistema Koppitz? Avaliação Psicológica, 5 (1), 13-22.

Britto, G. N. O. \& Santos, T. R. (1996). The Bender Gestalt test for 5-to 15-year old Brazilian children: norms and validity. Jornal de Medicina e Biologia, 29 (11), 1513-1518.

Chan, P. W. (2001). Comparison of visual motor development in Hong Kong and the USA assessed on the Qualitative Scoring System for the Modified Bender-Gestalt Test. Psychological Reports, 88 (1), 236-240.

Colorni, E. R. (1994). O teste gestáltico visomotor de Bender e o fracasso escolar: fator lesional ou emocional? Temas sobre Desenvolvimento, 4 (20), 20-24.

Koppitz, E. M. (1963). The Bender Gestalt Test for young children. New York: Grums Stratton.

Koppitz, E. M. (1975). The Bender Gestalt Test for Young Children. Traduzido para o Brasil em 1989 por R. N. Piccoli para a Editora Artes Médicas.

Koppitz, E. M. (1989). O Teste Gestáltico Bender para crianças. Porto Alegre: Artes Médicas.

Kroeff, P. (1992). Desempenho de crianças no teste de Bender e nível sócio-econômico-cultural. Psicologia: Reflexão e Crítica, 5 (2), 119-126. 
La Puente, M. \& Maciel Jr., J. (1984). Procedimentos operacionais na avaliação do Teste de Bender infantil. Estudos de Psicologia, 3/4, 76-92.

Machado, M. C. L. (1978). Uso do teste de Bender para avaliar a organização perceptivo-motora de escolares paulistas. Dissertação de Mestrado, PUC-SP, São Paulo.

Mazzeschi, C. \& Lis, A. (1999). The Bender-Gestalt Test: Koppitz Developmental Scoring System administered to two samples of italian preschool and primary school children. Perceptual and Motor Skills, 88, 1235-1244.

McCarron, L. M. \& Horn, P. W. (1979). Haptic visual discrimination and intelligence. Journal of Clinical Psychology, 35 (1), 117-120.

Neale, M. D. \& McKay, M. F. (1985). Scoring the Bender-Gestalt test using the Koppitz developmental system: interrater reliability, item difficulty, and scoring implications. Perceptual and Motor Skills, 60, 627-636.

Noronha, A. P. P.; Oliveira, A. F.; Cobêro, C.; Paula, L. M.; Cantalice, L. M.; Guerra, P. B. C.; Martins, R. M. M. \& Filizatti, R. (2002). Instrumentos psicológicos mais conhecidos por estudantes do sul de Minas Gerais. Avaliação Psicológica, 1 (2), 151-158.

Noronha, A. P. P. (2002). Os problemas mais graves e mais freqüentes no uso dos testes psicológicos. Psicologia: Reflexão $e$ Crítica, 15 (1), 135-142.

Pinelli Jr., B. \& Pasquali, L. (1991/1992). Parâmetros psicométricos do Teste Gestáltico Viso-Motor de Bender: um estudo empírico. Revista de Psicologia, 9/10 (1/2), 51-74.

Santucci, H. \& Galifret-Granjon, N. (1968). Prova Gráfica de Organização Perceptiva. Em R. Zazzo, Manual para o exame psicológico da criança (pp. 233-268). São Paulo: Editora Mestre Jou.
Silvestre, N., Salaverry, O. \& Gonzales, G. F. (1995). Madurez visomotora en escolares de ambos sexos de Lima $(150 \mathrm{~m})$ y de Cerro de Pasco (4340 m). Acta Andina, 4 (1), 35-42.

Sisto, F. F., Bueno, J. M. H. \& Rueda, F. J. M. (2003). Traços de personalidade na infância e distorção e integração de formas: um estudo de validade. Psicologia em Estudo, 8 (1), 77-84.

Sisto, F. F., Noronha, A. P. P. \& Santos, A. A. A. (2005). Bender Sistema de Pontuação Gradual B-SPG. São Paulo: Vetor Editora Psicopedagógica Ltda.

Sisto, F. F., Noronha, A. P. P. \& Santos, A. A. A. (2004). Distorção de forma no Teste de Bender: questionando seu critério de validade. Revista do Departamento de Psicologia da UFF, 16 (2), 139-154.

Sisto, F. F., Santos, A. A. A. \& Noronha, A. P. P. (2004). Critério de integração do Teste de Bender: explorando evidências de validade. Avaliação Psicológica, 3 (1), 13-20.

Suehiro, A. C. B. \& Santos, A. A. A. (2005). O Bender e as dificuldades de aprendizagem: estudo de validade. Avaliação Psicológica, 4 (1), 23-31.

Shapiro, S. K. \& Simpson, R. G. (1995). Koppitz scoring system as a measure of Bender-Gestalt performance in behaviorally asd emotionally disturbed adolescents. Journal of Clinical Psychology, 51 (1), 108-112.

Tosi, S. M. V. D. (1990). Adaptação Hutt do Teste Gestáltico de Bender em alcoólicos e toxicômanos. Temas, 38, 108-127.

Vance, B., Fuller, G. B. \& Lester, M. L. (1986). A comparison of the Minnesota Perceptual Diagnostic Test Revised and the Bender Gestalt. Jounal of Learning Disabilities, 19 (4), 211214.

Recebido: 15/08/2006 Revisado: 23/11/2006 Aceito: 20/12/2006

\section{Sobre as autoras:}

Adriana Cristina Boulhoça Suehiro: Psicóloga. Doutoranda em Psicologia pelo Programa de Pós-Gradução Stricto Sensu em Psicologia da Universidade São Francisco/Itatiba. Bolsista CAPES.

Acácia Aparecida Angeli dos Santos: Psicóloga. Doutora em Psicologia Escolar e Desenvolvimento Humano pela USP e Docente da Graduação e do Programa de Pós-Gradução Stricto Sensu em Psicologia da Universidade São Francisco/Itatiba. Bolsista produtividade do CNPq.

Endereço para correspondência: Dra. Acácia A. Angeli dos Santos - Universidade São Francisco - Programa de Pós-Graduação em Psicologia - R. Alexandre Rodrigues Barbosa, 45 - 13251-040 Itatiba - SP - Endereço eletrônico: acacia.santos@ saofrancisco.edu.br / acaciasantos@terra.com.br. 